\title{
One-thimble regularisation of lattice field theories: is it only a dream?
}

\author{
Francesco Di Renzo*, Simran Singh and Kevin Zambello \\ Dipartimento di Scienze Matematiche, Fisiche e Informatiche, Università di Parma and INFN, \\ Gruppo Collegato di Parma, I-43124 Parma, Italy \\ E-mail: francesco.direnzo@unipr.it, simran.singh@unipr.it, \\ kevin.zambello@studenti.unipr.it
}

Lefschetz thimbles regularisation of (lattice) field theories was put forward as a possible solution to the sign problem. Despite elegant and conceptually simple, it has many subtleties, a major one boiling down to a plain question: how many thimbles should we take into account? In the original formulation, a single thimble dominance hypothesis was put forward: in the thermodynamic limit, universality arguments could support a scenario in which the dominant thimble (associated to the global minimum of the action) captures the physical content of the field theory. We know by now many counterexamples and we have been pursuing multi-thimble simulations ourselves. Still, a single thimble regularisation would be the real breakthrough. We report on ongoing work aiming at a single thimble formulation of lattice field theories, in particular putting forward the proposal of performing Taylor expansions on the dominant thimble.

37th International Symposium on Lattice Field Theory - Lattice2019

16-22 June 2019

Wuhan, China

${ }^{*}$ Speaker. 


\section{Thimbles and single thimble dominance hypothesis in a nutshell}

The sign problem is a major obstacle to lattice simulations of theories we would be interested in, among which QCD at finite baryon density. The problem is quite easily described ${ }^{1}$ : we want to compute

$$
<O>=\frac{1}{Z} \int d x e^{-S(x)} O(x) \quad \text { with } S(x)=S_{R}(x)+i S_{I}(x)
$$

but (with a complex action in place) $e^{-S}$ can not be regarded as a decent (positive) probability measure and Monte Carlo simulations are not viable. Thimble regularisation [1,2] is easily described as well

- One complexifies the degrees of freedom, i.e. $x \rightarrow z=x+i y$ and $S(x) \rightarrow S(z)$.

- One then looks for critical points $p_{\sigma}$ where $\partial_{z} S=0$.

- The thimble $\mathscr{J}_{\sigma}$ attached to each critical point is the union of all the Steepest Ascent paths (SA) which are the solutions of $\frac{d}{d t} z_{i}=\frac{\partial \bar{S}}{\partial \bar{z}_{i}}$ stemming from the critical point (initial condition).

- Due to the olomorphic nature of $S$, the original integral is convergent on the thimble, with $S_{I}$ staying constant (so that the sign problem is killed).

Thimbles are manifolds of the same (real) dimension of the original manifold the theory was formulated on, but they are embedded in a manifold of twice that dimension. The integration measure on the thimble encodes the orientation of the latter with respect to the embedding manifold and this sadly reintroduces a residual sign problem due the so-called residual phase. We have no simple recipe to compute the integration measure other than the following one, which is easy to understand, but heavy to implement

- At the critical point one has to solve the Takagi problem for the Hessian of the action $H\left(S, p_{\sigma}\right) v^{(i)}=\lambda_{i} \bar{v}^{(i)}$.

- The Takagi values $\lambda_{i}$ fixe the rate at which the real part of the action increases along the SA paths.

- The Takagi vectors $v^{(i)}$ provide a basis for the tangent space at the critical point.

- The tangent space at each point on the thimble can be reconstructed by parallel transporting the Takagi vectors along the SA paths.

All in all, Lefschetz/Picard theory states that a thimble decomposition for the original integral holds

$$
<O>=\frac{\sum_{\sigma} n_{\sigma} e^{-i S_{I}\left(p_{\sigma}\right)} \int_{\mathscr{J}_{\sigma}} d z e^{-S_{R}} O e^{i \omega}}{\sum_{\sigma} n_{\sigma} e^{-i S_{I}\left(p_{\sigma}\right)} \int_{\mathscr{J} \sigma} d z e^{-S_{R}} e^{i \omega}}
$$

In (1.1) the harmless constant phase factors due to $S_{I}$ are factored in front of the integrals, while the residual sign problem is due to the residual phases $e^{i \omega}$. Both the numerator and the denominator

\footnotetext{
${ }^{1}$ In the following we adopt a light notation in which a field theory looks like an ordinary integral.
} 
(i.e. the partition function) receive contributions in principle by all the critical points, even if the intersection numbers $n_{\sigma}$ can be zero for possibly many critical points. Actually $n_{\sigma}=0$ for a critical point when the associated instable thimble does not intersect the original integration manifold ${ }^{2}$.

Collecting contributions from multiple thimbles can indeed make thimble regularisation a hard problem. Not only one has to deal with the computation of many contributions; the combination of many terms in the numerator (and denominator) of (1.1) can actually result in a renewed sign problem, given the multiple phase factors $e^{-i S_{I}\left(p_{\sigma}\right)}$. In the original proposal a single thimble dominance hypothesis was put forward. There could be situations in which the dominant thimble alone (i.e. the one associated to the absolute minimum of the real action) could encode the result one is interested in. A first argument is a very simple one: from semiclassical arguments, the contribution from the global minimum of the action is more and more enhanced in the thermodynamic limit. Moreover, universality arguments can be taken into account: the dominant thimble regularisation defines a local QFT with exactly the same symmetries, the same number of degrees of freedom (belonging to the same representations of the symmetry groups) and the same local interactions as the original theory. Moreover, the perturbative expansion is the same as in the original formulation. While these arguments are not enough to draw a definite conclusion, it was reassuring that in the first application of thimble regularisation (the relativistic Bose gas) the approximation proved to work very well [3].

However, it did not take that long for counterexamples to show up. In the case of the Thirring model it was shown that the dominant thimble could not capture the (complete) correct result $[4,5]$. This was one of the motivations for exploring alternative formulations inspired by thimbles. The idea of complexifing the degrees of freedom (in general, of deforming the original domain of integration) is in fact a very general one. Alternatives to thimbles appeared, e.g. the holomorfic flow [5] or various approached to the query for sign-optimised manifods, possibly enforced by deep-learning techniques $[6,7,8]$

\subsection{Multiple thimbles simulations}

Defining

$$
<O>_{\sigma}=\frac{\int_{\mathscr{J}_{\sigma}} d z e^{-S_{R}} O}{\int_{\mathscr{J}_{\sigma}} d z e^{-S_{R}}}=\frac{\int_{\mathscr{J}_{\sigma}} d z e^{-S_{R}} O}{Z_{\sigma}}
$$

we can rewrite (1.1) as

$$
<O>=\frac{\sum_{\sigma} n_{\sigma} e^{-i S_{I}\left(p_{\sigma}\right)} Z_{\sigma}<O e^{i \omega}>_{\sigma}}{\sum_{\sigma} n_{\sigma} e^{-i S_{I}\left(p_{\sigma}\right)} Z_{\sigma}<e^{i \omega}>_{\sigma}} .
$$

(1.2) has the obvious interpretation of an expectation value (VEV) on a single thimble, with the measure given by the real part of the action. (1.3) can in turns be intepreted as a weighted sum of $V E V$ contributions, the weights being given by coefficients involving the $Z_{\sigma}$. Stated in this way, the task of performing multiple thimbles simulations can be reduced to $(a)$ performing single thimble simulations; $(b)$ computing the relative weights in (1.3). As one could expect, it turns out that $(b)$ is a harder task than $(a)$. Nevertheless there were cases in which we could perform multiple thimbles simulations, according to two different strategies.

\footnotetext{
${ }^{2}$ The instable thimble is the union of the Steepest Descent (SD) paths stemming from a critical point.
} 
- There are cases in which it turns out that only a limited number of thimbles contributes, maybe also in presence of symmetries ensuring that a few contributions are equal. This turned out to be the case for QCD in $0+1$ dimensions [9], in which a successful computation came out of the sum of only two contributions (this is just a case in which a symmetry is in place) according to

$$
<O>=\frac{<O e^{i \omega}>_{\sigma_{1}}+\alpha<O e^{i \omega}>_{\sigma_{2}}}{<e^{i \omega}>_{\sigma_{1}}+\alpha<e^{i \omega}>_{\sigma_{2}}} .
$$

Here the idea is to rewrite (1.3) putting all our ignorance into a single parameter and giving up hope of a first principles derivation of relative weights. One should instead fix the value of $\alpha$ assuming one known measurement as a normalization point and then predicting the value of other observables. Something similar can be put at work in the playground that first revealed the failure of single thimble simulations, i.e. the Thirring model [10].

- Relative weights can in turns be computed in a semiclassical approximation (according to what is also referred to as the gaussian approximation). A possible strategy is to start with this semiclassical computation and then compute corrections to it. It turned out that this works pretty well in the context of a minimal version of the so-called Heavy Dense approximation for QCD [11].

All in all, we were able to show that some steps can be taken in the direction of multiple thimbles simulations. The path to success is nevertheless a difficult one and in the end the real breakthrough would be going back to the idea of (some form of) one thimble simulation.

\section{Thimble decomposition and Stokes phenomena}

In order to gain some understanding on the thimble decomposition, one should consider the situations in which it fails. This is when a Stokes phenomenon occurs. A Stokes phenomenon takes place when two different critical points are connected by a SA/SD path. This simply means that the stable thimble of one critical point sits on top of the unstable thimble of another one. Under this conditions there is no thimble decomposition. It turns out that changes in the thimble decomposition can be traced back to the occurence of Stokes phenomena: a very effective description of all this can be found in [12] (just in the case of the Thirring model). A semplified, intuitive picture of the relationship between thimble decomposition and Stokes phenomena can be given as in the following.

- A thimble decomposition is in place when the union of a given number of thimbles is essentially a deformation of the original integration contour, just like in applications of Cauchy theorem (this was just the spirit of [5]).

- As they are solutions of the same differential equation subject to different initial conditions, different thimbles can not cross each other. This in turns means that they act as barriers to each other in the thimble decomposition: when the union of a given number of thimbles is a correct deformation of the original integration contour, other thimbles are simply kept out. 
- Moving around in the parameter space of a given theory, thimbles do move around in the manifold embedding the original one, but they do it smoothly, i.e. they are always subject to the constraint of not crossing each other. Thus the thimbles that contribute to the decomposition of the original domain of integration keep on keeping the others out.

- There is only one way thimbles can cross each other and this is just when two thimbles sit on top of each other (two different critical points are connected by a SA/SD path and the stable thimble of one sits on top of the unstable thimble of the other). When this occurs we are in presence of a Stokes phenomenon and thimble decomposition fails.

- After a Stokes phenomenon has occured, the relative arrangement of thimbles can change and a different thimble decomposition is in place.

\section{Taylor expansions on Lefschetz thimbles}

A very important point we want to stress is that Stokes phenomena mark discontinuities in the thimble decomposition, i.e. in the coefficients $n_{\sigma}$. This does not mean that physical observables are discontinuous. Continuity of physical observables across discontinuities of the $n_{\sigma}$ coefficients (i.e. of the thimble decomposition) is indeed a possible handle on fixing the values of the $n_{\sigma}$ themselves ${ }^{3}$. This is not the end of the story, and the continuity of physical observables across Stokes phenomena has yet another interesting application.

The idea is to Taylor expand an observable around a point $\mu_{0}$ where one single thimble is enough to reconstruct the correct result

$$
\langle O\rangle(\mu)=\langle O\rangle\left(\mu_{0}\right)+\left.\frac{\partial\langle O\rangle}{\partial \mu}\right|_{\mu_{0}}\left(\mu-\mu_{0}\right)+\left.\frac{1}{2} \frac{\partial^{2}\langle O\rangle}{\partial \mu^{2}}\right|_{\mu_{0}}\left(\mu-\mu_{0}\right)^{2}+\ldots
$$

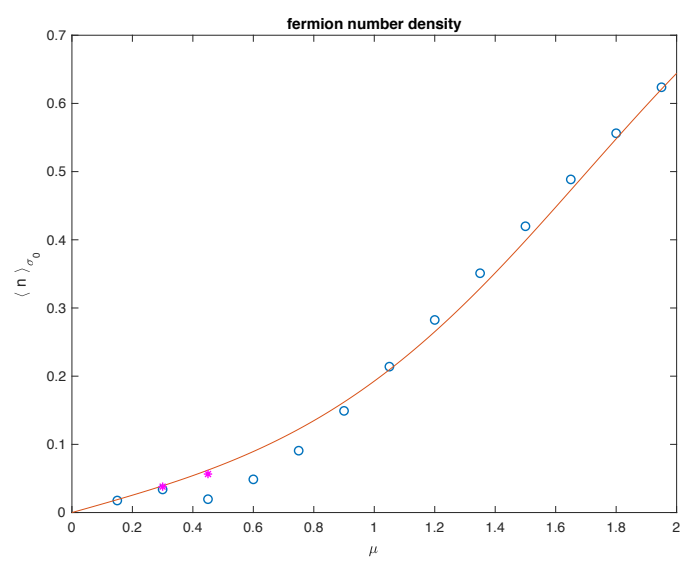

Figure 1: The fermion number density for the $L=2, \beta=1, m=1$ Thirring model. The solid line is the (known) exact result. Circles are the result of a single thimble computation. Stars are the result of the Taylor expansion.

\footnotetext{
${ }^{3}$ See the discussion of the simple $\phi^{4}$ toy model in [13].
} 
All in all, we compute Taylor expansion coefficients on a single thimble in a region in which this is enough and then we reconstruct values of the observable in a region in which multiple thimbles would be needed to reconstruct the correct result.

A toy model application is displayed in Figure 1: this is the computation of the fermion number density for the $L=2$ Thirring model $(\beta=1, m=1)$. The solid line is the (known) exact result. Circles are the result of a single thimble computation: as one can see, in an extended region this fails to reconstruct the correct result. Stars are the result of the Taylor expansion (i.e. a few steps taken into the bad region by computing Taylor coefficients in the good one). A few more details can be found in [10].

This is not the end of the story. After the conference we were able to show that by bridging different regions in which one single thimble is enough to evaluate Taylor coefficients, one can in some cases circumvent the necessity of performing multiple thimbles simulations.

\section{Conclusions}

The ideas that were discussed at the conference were admittedly very prototypal ones and only a very basic example of performing Taylor expansions on Lefschetz thimbles was provided. After the conference we made some progress, which will be the subject of a paper which will be issued soon. All in all, we are confident that the idea of having Taylor expansions bridging regions in which single thimble simulations are correct can indeed (in some cases) circumvent the necessity of multiple thimble simulations.

\section{Acknowledgements}

This work has received funding from the European Union's Horizon 2020 research and innovation programme under the Marie Skłodowska-Curie grant agreement No. 813942 (EuroPLEx). We also acknowledge support from I.N.F.N. under the research project i.s. QCDLAT.

\section{References}

[1] M. Cristoforetti et al. [AuroraScience Collaboration], New approach to the sign problem in quantum field theories: High density QCD on a Lefschetz thimble, Phys. Rev. D 86 (2012) 074506 [arXiv:1205.3996 [hep-lat]].

[2] H. Fujii, D. Honda, M. Kato, Y. Kikukawa, S. Komatsu and T. Sano, Hybrid Monte Carlo on Lefschetz thimbles - A study of the residual sign problem, JHEP 1310 (2013) 147 [arXiv:1309.4371 [hep-lat]].

[3] M. Cristoforetti, F. Di Renzo, A. Mukherjee and L. Scorzato, Monte Carlo simulations on the Lefschetz thimble: Taming the sign problem, Phys. Rev. D 88, no. 5, 051501 (2013) doi:10.1103/PhysRevD.88.051501 [arXiv:1303.7204 [hep-lat]].

[4] H. Fujii, S. Kamata and Y. Kikukawa, Monte Carlo study of Lefschetz thimble structure in one-dimensional Thirring model at finite density, JHEP 1512 (2015) 125 Erratum: [JHEP 1609 (2016) 172] doi:10.1007/JHEP12(2015)125, 10.1007/JHEP09(2016)172 [arXiv:1509.09141 [hep-lat]].

[5] A. Alexandru, G. Basar, P. F. Bedaque, G. W. Ridgway and N. C. Warrington, Sign problem and Monte Carlo calculations beyond Lefschetz thimbles, JHEP 1605, 053 (2016) [arXiv:1512.08764 [hep-lat]]. 
[6] A. Alexandru, P. F. Bedaque, H. Lamm and S. Lawrence, Deep Learning Beyond Lefschetz Thimbles, Phys. Rev. D 96, no. 9, 094505 (2017) doi:10.1103/PhysRevD.96.094505 [arXiv:1709.01971 [hep-lat]].

[7] A. Alexandru, P. F. Bedaque, H. Lamm and S. Lawrence, Finite-Density Monte Carlo Calculations on Sign-Optimized Manifolds, Phys. Rev. D 97, no. 9, 094510 (2018) doi:10.1103/PhysRevD.97.094510 [arXiv:1804.00697 [hep-lat]].

[8] Y. Mori, K. Kashiwa and A. Ohnishi, Toward solving the sign problem with path optimization method, Phys. Rev. D 96, no. 11, 111501 (2017) doi:10.1103/PhysRevD.96.111501 [arXiv:1705.05605 [hep-lat]].

[9] F. Di Renzo and G. Eruzzi, One-dimensional QCD in thimble regularization, Phys. Rev. D 97, no. 1, 014503 (2018) doi:10.1103/PhysRevD.97.014503 [arXiv:1709.10468 [hep-lat]].

[10] K. Zambello and F. Di Renzo, On the Lefschetz thimbles structure of the Thirring model, PoS LATTICE 2019, 211 [arXiv:1912.11380 [hep-lat]].

[11] K. Zambello and F. Di Renzo, Towards Lefschetz thimbles regularization of heavy-dense QCD, PoS LATTICE 2018, 148 (2018) doi:10.22323/1.334.0148 [arXiv:1811.03605 [hep-lat]].

[12] H. Fujii, S. Kamata and Y. Kikukawa, Lefschetz thimble structure in one-dimensional lattice Thirring model at finite density, JHEP 1511 (2015) 078 Erratum: [JHEP 1602 (2016) 036] doi:10.1007/JHEP02(2016)036, 10.1007/JHEP11(2015)078 [arXiv:1509.08176 [hep-lat]].

[13] F. Di Renzo and G. Eruzzi, Thimble regularization at work: from toy models to chiral random matrix theories, Phys. Rev. D 92, no. 8, 085030 (2015) doi:10.1103/PhysRevD.92.085030 [arXiv:1507.03858 [hep-lat]]. 